\title{
P I 9-55 LB. Effective control of a pathogenic SIVmac239 challenge by a novel heterologous mucosal prime and intramuscular boost vaccine strategy
} Z Chen*1, C Sun ${ }^{2}, \mathrm{Y} \mathrm{Du}^{1}{ }^{2}$ L Chen ${ }^{2}$ and L Zhang ${ }^{3}$

Address: ${ }^{1}$ AIDS Institute, The University of Hong Kong LKS Faculty of Medicine, Hong Kong, PR China, ${ }^{2}$ Guangzhou Institute of Biomedicine and Health, Chinese Academy of Scie, Guanzhou, Guangdong, PR China and ${ }^{3}$ Comprehensive AIDS Research Center, Tsinghua University; AIDS Research, Beijing, PR China

* Corresponding author

from AIDS Vaccine 2009

Paris, France. 19-22 October 2009

Published: 22 October 2009

Retrovirology 2009, 6(Suppl 3):P4I9 doi:10.1186/1742-4690-6-S3-P4I9

This abstract is available from: http://www.retrovirology.com/content/6/S3/P4I9

(c) 2009 Chen et al; licensee BioMed Central Ltd.

\section{Background}

The failure of a recombinant adenovirus serotype 5 (rAd5) vector-based vaccine for HIV-1 in a phase $2 \mathrm{~b}$ efficacy study in humans calls for efforts to develop novel vaccination strategies.

\section{Methods}

In this study, we developed a recombinant replicationcompetent modified vaccinia Tiantan (MVTT), namely $\mathrm{rMVTT}_{\text {SIVgpe }^{\prime}}$ as a mucosal vaccine expressing SIVmac Gag, Pol and Env. The immunogenicity and efficacy of rMVTT $_{\text {SIVgpe }}$ was studied in combination with an rAd5based vaccine rAd5 $5_{\text {sIVgpe }}$ in Chinese macaques (Macaca mulatta) without the protective MHC class I allele Mamu$\mathrm{A}^{*} 01$. $\mathrm{rMVTT}_{\text {SIVgpe }}$ was given through intranasal and oral inoculations whereas $\mathrm{rAd} 5_{\text {SIVge }}$ was given through intramuscular injection. Four macaques in each of the four study groups received the following prime and boost vaccinations: $\mathrm{rMVTT}_{\text {SIVge }} / \mathrm{rAd}_{\text {SIVgpe }} ; \mathrm{rMVTT}_{\text {SIVgpe }} / \mathrm{rAd}_{\text {SIVgpe }^{\prime}}$ twice; rAd5 $5_{\text {SIVgpe }} / \mathrm{rAd} 5_{\text {SIVgpe }}$ and placebo controls, respectively.

\section{Results}

We found that the heterologous $\mathrm{rMVTT}_{\text {SIVgpe }} / \mathrm{rAd}_{\text {SIVge }}$ regimen elicited cellular immune responses with enhanced magnitude, breadth, sustainability, and polyfunctionality when compared with the homologous rAd5 $5_{\text {SIVgpe }}$ regimen. Higher levels of neutralizing antibody $(\mathrm{Nab})$ responses were also induced by the $\mathrm{rMVTT}_{\text {SIVgpe }}$
rAd5 $5_{\text {SIVgpe }}$ regimen. These Nab responses, however, neutralized SIVmac1A11 but not SIVmac239. The additional round of $\mathrm{rMVTT}_{\text {SIVgpe }} / \mathrm{rAd}_{\text {SIVge }}$ vaccinations did not enhance the immune responses further. After intrarectal challenge with a pathogenic and Chinese macaqueadapted SIVmac239 (5 × $10^{5}$ TCID $_{50}$ per animal), one of four monkeys vaccinated with the $\mathrm{rMVTT}_{\text {SIVgpe }} / \mathrm{rAd} 5_{\text {SIVgpe }}$ regimen was fully protected whereas the rest showed an average of $1.96 \mathrm{log}$ and $2.22 \mathrm{log}$ reduction of peak and setpoint ( 6 weeks post challenge) viral loads as compared with control animals.

\section{Conclusion}

These data demonstrate that the $\mathrm{rMVTT}_{\text {SIVgpe }} / \mathrm{rAd} 5_{\text {SIVge }}$ regimen induced durable partial immune control of a pathogenic, neutralization-resistant SIVmac239 challenge. Our findings have critical implications for further optimization of vaccination strategies against HIV-1 by engaging the mucosal immune system. 